\title{
Role of terminal and anastomotic circulation in the patency of arteries jailed by flow-diverting stents: animal flow model evaluation and preliminary results
}

\author{
Christina losif, MD, MSc, PhD, ${ }^{1,2}$ Philipp Berg, PhD, ${ }^{3}$ Sebastien Ponsonnard, MD, ${ }^{4}$ \\ Pierre Carles, RE, ${ }^{5}$ Suzana Saleme, MD, ${ }^{1}$ Eduardo Pedrolo-Silveira, MD,, Georges Mendes, MD,1 \\ Eduardo Waihrich, MD, ${ }^{1}$ Gilles Trolliard, PhD, ${ }^{5}$ Claude-Yves Couquet, DVM, ${ }^{6}$ \\ Catherine Yardin, MD, PhD, 2,7 and Charbel Mounayer, MD, PhD ${ }^{1,2}$
}

\begin{abstract}
Departments of ${ }^{1}$ Interventional Neuroradiology and ${ }^{4}$ Anesthesiology, Dupuytren University Hospital (CHU Limoges); ${ }^{2}$ Applied Medical Research Team (ERMA); ${ }^{5}$ Science of Ceramic Processes and Surface Treatments, CNRS, UMR 7315, European Ceramic Center, University of Limoges; ${ }^{6}$ Research and Analysis Laboratory, Haute-Vienne; 'Department of Histology, Cytology, Cellular Biology and Cytogenetics, Mother and Child (HME) University Hospital, Limoges, France; and 'Laboratory of Fluid Dynamics and Technical Flows, University of Magdeburg "Otto von Guerike," Magdeburg, Germany
\end{abstract}

\begin{abstract}
OBJECTIVE The authors describe herein the creation of an animal model capable of producing quantifiable data regarding blood flow rate and velocity modifications in terminal and anastomotic types of cerebrofacial circulation. They also present the preliminary results of a translational study aimed at investigating the role of terminal and anastomotic types of circulation in arterial branches jailed by flow-diverting stents as factors contributing to arterial patency or occlusion.

METHODS Two Large White swine were used to validate a terminal-type arterial model at the level of the right ascending pharyngeal artery (APhA), created exclusively by endovascular means. Subsequently 4 Large White swine, allocated to 2 groups corresponding to the presence (Group B) or absence (Group A) of terminal-type flow modification, underwent placement of flow-diverting stents. Blood flow rates and velocities were quantified using a dedicated time-resolved 3D phase-contrast MRA sequence before and after stenting. Three months after stent placement, the stented arteries were evaluated with digital subtraction angiography (DSA) and scanning electron microscopy (SEM). Patent (circulating) ostia quantification was performed on the SEM images.
\end{abstract}

RESULTS Terminal-type flow modification was feasible; an increase of $75.8 \%$ in mean blood velocities was observed in the right APhAs. The mean blood flow rate for Group A was $0.31 \pm 0.19 \mathrm{ml} / \mathrm{sec}(95 \% \mathrm{Cl}-1.39$ to 2.01) before stenting and $0.21 \pm 0.07 \mathrm{ml} / \mathrm{sec}(95 \% \mathrm{Cl}-0.45$ to 0.87$)$ after stenting. The mean blood flow rate for Group B was $0.87 \pm 0.32 \mathrm{ml} /$ $\sec (95 \% \mathrm{Cl}-1.98$ to 3.73$)$ before stenting and $0.76 \pm 0.13 \mathrm{ml} / \mathrm{sec}(95 \% \mathrm{Cl}-0.41$ to 1.93$)$ after stenting. Mean flow rates after stenting showed a statistically significant difference between Groups A and B (Welch test). Mean and maximal blood velocities were reduced in Group A cases and did not decrease in Group B cases. Control DSA and SEM findings showed near occlusion of the jailed APhAs in both cases of anastomotic circulation (mean patent ostium surface 32,776 $\mu \mathrm{m}^{2}$ ) and patency in both cases of terminal-type circulation (mean patent ostium surface $422,334 \mu \mathrm{m}^{2}$ ).

CONCLUSIONS Terminal-type arterial modification in swine APhAs is feasible. Sufficient data were acquired to perform an a priori analysis for further research. Flow diversion at the level of the APhA ostium resulted in significant stenosis in cases of anastomotic circulation, while sufficient patency was observed in terminal-type circulation.

http://thejns.org/doi/abs/10.3171/2015.8.JNS151296

KEY WORDS flow diverting stent; collateral arteries; endothelialization; ostium; embolization; magnetic resonance angiography; vascular disorders

ABBREVIATIONS APhA = ascending pharyngeal artery; $C C A=$ common carotid artery; $C F D=$ computational fluid dynamics; $D S A=$ digital subtraction angiography; $E C A$ = external carotid artery; FDS = flow-diverting stent; pCMRA = phase contrast MR angiography; $P E D=$ Pipeline embolization device; RM = rete mirabile; $S E M=$ scanning electron microscopy.

SUBMITTED June 5, 2015. ACCEPTED August 5, 2015.

INCLUDE WHEN CITING Published online January 15, 2016; DOI: 10.3171/2015.8.JNS151296. 
$\mathrm{F}$ LOW-DIVERTING stents (FDSs) represent a very interesting alternative for the treatment of anatomically challenging intracranial aneurysms. ${ }^{1}$ Initial animal studies in rabbits showed patency of arterial branches jailed by this device. ${ }^{18}$ Nevertheless, recent clinical data challenge this conclusion, ${ }^{5}$ even in adequately premedicated patients. ${ }^{20}$

Clinical observations led us to assume that the presence of a direct collateral supply for a territory supplied by an intracranial artery favors asymptomatic occlusion of that artery when it is jailed by an FDS. ${ }^{12}$ On the contrary, when only pial collateral circulation is present, the arterial branch tends to remain patent, without significant remodeling over time. ${ }^{25}$ To date these observations have not been exhaustively explored qualitatively and quantitatively. In a simplified model with straight arterial segments and minimal stent pore variations, we expect these phenomena to be reproduced in terminal and anastomotic types of circulation. In a terminal type of circulation, no significant reduction in blood velocities and flow rates is expected in the covered artery poststenting. On the contrary, in the presence of collateral supply at an anatomically similar arterial segment, blood flow rates and velocities through the stent are expected to significantly decrease. These phenomena are expected to affect the vascular remodeling process and endothelialization rate on the free segments of the stents.

In this study we attempted to simulate terminal and anastomotic types of circulation in arteries having size and hemodynamic characteristics similar to those in proximal intracranial branches, namely diameters of 1-2 $\mathrm{mm}$ and positive end-diastolic waveform curves. Our goals were to evaluate the modifications in blood flow rates, velocities, and pressure gradients induced by the stent apposition and, finally, to correlate them with the imaging and anatomopathological results after a chronological period adequate for sufficient neointimal development on the stent-covered arterial ostia.

\section{Methods}

A translational animal study on Large White swine (6 total) was designed, including a preliminary phase of a flow model evaluation, a feasibility study, and a preliminary results evaluation. Four swine, 2 male and 2 female, were 3 months old on the day of stenting. Mean body weight on the day of stenting was $20.5 \pm 1.1 \mathrm{~kg}$. Animals used for the flow model evaluation were of similar age and weight.

The anastomotic type of circulation or the presence of flow competition regarding the arterial branch explored was defined as the presence of a significant, direct collateral arterial network contributing to the supply of this branch's vascular territory (Group A). The terminal type of circulation or the absence of flow competition was defined as the absence of a significant collateral supply for the branch's vascular territory (Group B).

\section{Ethical Standards and Animal Care}

The institutional ethics committee for animal care and the regional ethics committee for experiments on animals approved this study and validated its adherence to the national guidelines and regulations for the care and use of laboratory animals. The study design and reporting are in accordance with the ARRIVE guidelines ${ }^{19}$ for reporting animal research. Animal care installations, anti-aggregation protocol, and general care were identical for all animals and adhered to the European Convention for the Protection of Vertebrate Animals Used for Experimental and Other Scientific Purposes. ${ }^{22}$

\section{Blinding Methods}

Animals were randomized to 2 groups via a closed envelope containing the identification number of each animal. Concealment of group allocation was constant throughout the experimental period, from group randomization until euthanasia. Blinding during the interpretation and statistical analysis of quantitative data was applied as well.

\section{Anesthesia, Analgesia, Medications, and Euthanasia}

Animals were premedicated with aspirin $(10 \mathrm{mg} / \mathrm{kg}$ orally) and clopidogrel (10 mg/kg orally) 48 hours prior to intervention, a regimen that was maintained throughout the follow-up period of 3 months. Endovascular procedures and MRI were performed while each animal was under general anesthesia. Each animal was premedicated with intramuscular ketamine $(20 \mathrm{mg} / \mathrm{kg})$ and xylazine $(2$ $\mathrm{mg} / \mathrm{kg}$ ) and intubated. Anesthesia was maintained with propofol and sevoflurane. ${ }^{9}$ The Seldinger technique was performed via a percutaneous right femoral approach for all interventions, during which a local percutaneous anesthetic (lidocaine) was used. At 3 months poststenting, animals were euthanized with barbiturate overdose $(390 \mathrm{mg}$ sodium pentobarbital and $50 \mathrm{mg} / \mathrm{ml}$ sodium phenytoin, $0.22 \mathrm{ml} / \mathrm{kg}$ intravenously) after they had been anesthetized with propofol.

\section{Flow Model Rationale and Creation}

The Large White swine was chosen as an experimental animal because of the similarity of its ascending pharyngeal arteries (APhAs) with human intracranial arteries in terms of diameter (1-2 mm) and blood waveforms. ${ }^{23}$ The swine model has a complex cerebrofacial anatomy that allows for the creation of a sufficient "sump effect" in its APhAs with the exploitation of the rostral epidural rete mirabile, or simply rete mirabile (RM) ${ }^{24}$ a dense arterial network at the distal end of the APhAs at the skull base, through which the brain carotid arteries are supplied (Fig. 1).

By selective endovascular embolization of the anastomotic branches (ramus anastomoticus, arteria anastomotica), which connect the RM with the internal maxillary artery (cervicofacial supply), intracranial supply to the brain carotid arteries was maintained via the APhAs of both sides through the RM. By further endovascular coil obliteration of the left APhA, vascularization of the RM by only the right $\mathrm{APhA}$ was achieved. This corresponds to a terminal-type of arterial circulation regarding the RM.

\section{Endovascular Procedures}

All endovascular procedures and follow-ups were per- 


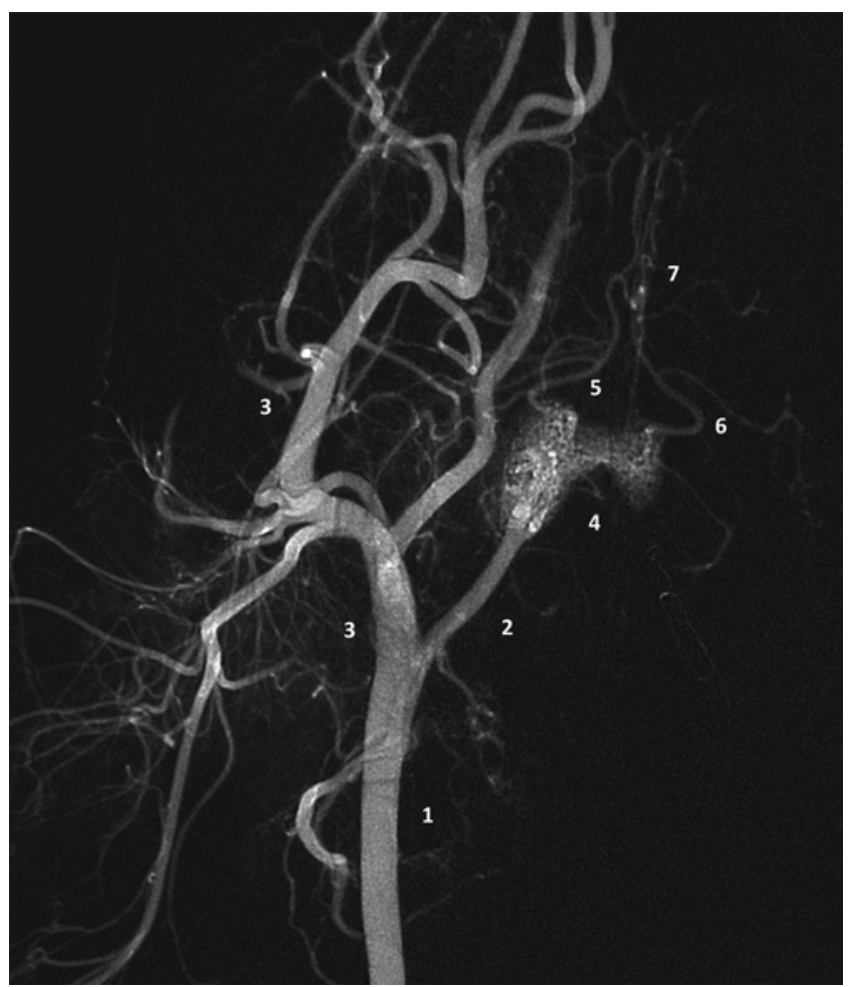

FIG. 1. Right common carotid artery (CCA) injection digital subtraction angiogram, anteroposterior view, showing the craniofacial arterial anatomy of the Large White swine. $1=\mathrm{CCA} ; 2=\mathrm{APhA}$, the equivalent of the internal carotid artery (ICA) in humans in terms of intracranial supply; $3=$ external carotid artery (ECA) with its branches, supplying the snout (rostrum), tongue, pinnae, eyes, and surrounding tissues of the head, as well as the anatomical structures of the neck; $4=$ rostral epidural rete mirabile (RM), 2 lobes interconnected with an isthmus of arterial anastomotic network at the midline, giving rise to the 2 brain carotid arteries (right and left), which in turn branch to supply the brain; $5=$ right brain carotid artery; $6=$ rostral branch of the left middle cerebral artery (MCA; left and right); 7 = rostral cerebral arteries (left and right).

formed on a biplane, flat-panel digital subtraction angiography (DSA) unit (Allura Xper FD20, Philips). Arterial diameters for stent size choice were measured with contrastenhanced 3D rotational angiography (contrast medium injection rate $4 \mathrm{ml} / \mathrm{sec}$ and volume $16 \mathrm{ml}$, delay of $1 \mathrm{sec}$ ). In all cases the nonionic iodinated contrast medium (300 $\mathrm{mg} / \mathrm{ml}$ Iohexol, Omnipaque, GE Healthcare) was administered through a preprogrammed mechanical injector.

\section{Flow Model Endovascular Modifications}

The left APhAs were occluded by means of bare platinum coils with diameters ranging from 2 to $4 \mathrm{~mm}$. Anastomotic branches to the RM were successfully occluded with a $50 \%$ mixture of $\mathrm{N}$-butyl-cyanoacrylate glue (Glubran, GEM Srl) and ethiodized oil (Lipiodol, André Guerbet) without adverse events.

\section{Main Study Protocol}

Four Large White swine were allocated to 2 groups (A and B) of 2 animals. Animals of Group A did not undergo cerebrofacial flow modification, whereas those of Group B underwent cerebrofacial arterial modification, as de- scribed above. Thus, Group A corresponded to the anastomotic type of circulation and Group B represented the terminal type.

A 6-Fr guiding catheter (Navien, Medtronic) was navigated into the common carotid artery (CCA), and the delivery microcatheter (Marksman, Medtronic) was positioned in the right external carotid artery (ECA). The stent (Pipeline embolization device [PED], Medtronic) with a nominal diameter was chosen in all cases, and its length was consistently $20 \mathrm{~mm}$. The stent was deployed to cover the right $\mathrm{APhA}$ ostium at its middle part. Before and after stenting, direct pressure measurements in the CCA and ECA were obtained through the microcatheter. Before stenting, the APhA pressure measurements were also obtained through the microcatheter. Poststenting, however, $\mathrm{APhA}$ pressure measurements were indirectly calculated using computational fluid dynamics (CFD) to avoid the influence of a second jailed microcatheter at the pressure difference measurements before and after stenting.

\section{Velocity and Flow Rate Evaluation Techniques}

Before and after selective endovascular arterial occlusions, blood velocities and flow rates at the level of the right $\mathrm{APhA}$ were assessed using color Doppler ultrasonography (Aplio-400, Toshiba Medical Systems Corp.) with a 10$\mathrm{MHz}$ probe (angle $26^{\circ}$ ). Velocity waveforms were obtained.

For the main experiments, thus before and after stenting, as well as at the 3-month follow-up, mean flow rates and velocities were measured using a time-resolved 3D phase-contrast MR angiography (pcMRA) protocol. This protocol was not used for the model evaluation since color Doppler ultrasonography with the previously described standardized protocol was sufficient to evaluate the velocity modifications; ${ }^{16}$ the more resource-consuming, but very accurate, MRA protocol was not necessary for this part of the study.

Time-resolved 3D pcMRA data were obtained on a 3.0-T system (Achieva, Philips Healthcare) using a 16-channel neurovascular coil (SENSE-NV-16) with retrospective electrocardiographic gating. The scanning parameters were as follows: FOV $230 \times 188.5 \times 40.5 \mathrm{~mm}$, reconstruction matrix $352 \times 352$, ACQ voxel MPS $(\mathrm{mm})$ 0.80/0.86/0.90, REC voxel MPS (mm) 0.65/0.65/0.90, acquisition matrix MXP $288 \times 219$, TR $8.3 \mathrm{msec}$, TE 4.5 msec, $\alpha=15^{\circ}$, number of excitations 1 , and receiver bandwidth $\pm 64.8 \mathrm{kHz}$. Flow velocity encoding (Venc) of 70 $\mathrm{cm} / \mathrm{sec}$ was chosen along each of the 3 principal axes. The data were reconstructed at 14 time points evenly spaced over the R-R interval (cardiac cycle). Total acquisition times for 4D flow MRI were heart rate-dependent and ranged between 18 and 25 minutes.

A diffusion-weighted sequence was added at the end of each MRI examination to search for embolic or ischemic events poststenting and at the follow-up. Diffusion gradients were applied in each of the $\mathrm{x}, \mathrm{y}$, and $\mathrm{z}$ directions with two b values $\left(0\right.$ and $\left.1000 \mathrm{sec} / \mathrm{mm}^{2}\right)$.

\section{Computational Fluid Dynamics}

Case-specific blood flow simulations were performed, including a pre- and poststenting simulation for each data 
set to evaluate the impact of the flow-diverting device. The overall computational workflow included 5 steps. 1) Vascular reconstruction: For each subject a 3D vessel model was reconstructed based on the acquired 3D rotational angiography images. For this purpose MeVisLab 2.3 (MeVis Medical Solutions AG) was used for medical image processing and visualization. Inflow and outflow crosssections were sufficiently extruded to guarantee fully developed and stable flow conditions. 2) Device modeling: An undeformed PED was virtually reproduced based on the implanted stent that was used during every intervention. Device length and diameter, strut diameter, and pore angles were adjusted accordingly. An in-house software package $^{2}$ was used to virtually deploy the stents in the corresponding vessel section. 3) Spatial discretization: To be able to compare the prestenting flow results with the virtually deployed configurations, volume meshes were generated again. Explicit description of the flow-diverting devices required volume element numbers ranging from 10 to 15 million. 4) Hemodynamic simulations: The hemodynamic flow behavior within the considered vessel regions was modeled using 3D Navier-Stokes equations. In each subject flow measurements were obtained before and after stent deployment by using the time-resolved 3D pcMRA measurements. Hence, the time-dependent flow curves for the main vessels (CCA, ECA, and APhA) were applied to the corresponding cross-sections. Vessel and stent walls were assumed to be rigid, and blood was treated as an incompressible $\left(\rho=1055 \mathrm{~kg} / \mathrm{m}^{3}\right)$ Newtonian $(\eta=4 \mathrm{mPa} \cdot \mathrm{s})$ fluid. For the remaining outlets the flow was split depending on the corresponding surface area. The high spatial and temporal $\left(\Delta \mathrm{t}=1 \cdot 10^{-4} \mathrm{sec}\right)$ resolutions and the consideration of at least 2 cardiac cycles lead to simulations lasting for several days per case. 5) Qualitative and quantitative analysis: To evaluate the impact of the virtual PED deployment on local hemodynamics, isosurface velocity contours were generated, providing visualization of the local flow structures and identifying regions of velocity reduction. Furthermore, velocity cut planes were compared pre- and poststenting to describe the flow behavior close to the covered vessel ostium. Detailed information regarding the CFD protocol has been published elsewhere. 3,14,15

\section{A Posteriori Evaluation of CFD Simulations}

Two researchers (C.I. and C.M.) independently performed a qualitative assessment by comparing the cutplane velocity simulations before and after stenting with 30-images/sec DSA runs at working projections before and after stenting, for all cases. Furthermore the free segments of stent, photographed using scanning electron microscopy (SEM) at 3 months, were correlated with the images of the virtual stenting. Appropriate software (ImageJ1, National Institutes of Health) was used to measure 4 stent strut angles on posteriorly illuminated optical microscopic images (Nikon Eclipse 50i microscope, Nikon Inc.), and these angles were compared with those produced by the virtual stenting for each stented ostium. Variations in angles from $0^{\circ}$ to $9^{\circ}$ were considered acceptable.

\section{Scanning Electron Microscopy}

Three months after stent placement the stented arter- ies were harvested en bloc and longitudinally opened to expose the inner surface of the stented $\mathrm{APhA}$ ostia, which were observed with SEM (JSM-7400F scanning electron microscope, JEOL). The specific chemical fixation and SEM preparation protocols are described in detail elsewhere. ${ }^{13}$ Digital images were taken (voltage $10-15 \mathrm{kV}$, magnifications $\times 35$ to $\times 120$ ), allowing us to observe ostia ranging from $3 \mathrm{~mm}$ to $50 \mu \mathrm{m}$. Quantification of the endothelialized ostia surfaces was performed with the ImageJ1 open-source software.

\section{Study Feasibility and Experimental Outcomes}

Animal immobility during the stenting and MRI manipulations was evaluated using an in-house 4-step scale. A veterinarian who was blinded to the animal group assignments assessed the clinical state and vital signs of the animals. In the flow model validation, the primary end point was an increase $\geq 40 \%$ in mean velocity values inside the right APhA post-flow modification. The secondary end point was a mean velocity value at least equal to the postocclusion velocities 3 weeks post-endovascular modifications. ${ }^{10}$ In the preliminary results validation, qualitative analysis of the DSA runs before and after stenting was performed as regards the jailed APhAs. Mean flow rates and maximal velocities before and after stenting were compared between the 2 groups. Quantitative evaluation of the stented ostia was performed using the SEM images of the longitudinally opened arterial specimen.

\section{Statistical Methods}

Flow rates, velocities, and ostia surfaces were treated with descriptive statistics and are expressed as the means and standard deviations and 95\% confidence intervals of the mean. Comparisons of mean flow rates and ostia surfaces between Groups A and B were done, assuming unequal variances, by means of the Welch test. Power analysis was performed using the mean and standard deviation of mean flow rates before and after stenting, and effect size estimation regarding the patent (circulating) ostia surfaces was done using Statistica software (StatSoft Inc.). The level of statistical significance was $<0.05$.

\section{Results}

All animals maintained normal weight gain curves, and veterinary evaluation revealed that all animals were in satisfactory condition throughout the follow-up period. Given the absence of adverse events, no modification of the study protocol was judged necessary.

\section{Technical Feasibility}

Each of the 4 stenting procedures, including endovascular stenting and MRI sequences before and after stenting, lasted for $134 \pm 8.4$ minutes. General anesthesia was feasible without movement from the animals ( 1 case scored 1 and all others scored 0 on the in-house movement scale) or any compromise in their general state. Immobility was very important for the time-resolved 3D pcMRA acquisitions and it was achieved without exception. All MRI 
acquisitions were free of movement artifacts and fully exploitable. The mean waking time for the animals after an intervention was $20 \pm 3.1$ minutes and was uneventful in all cases.

\section{Animal Model Validation}

Both animals used for the evaluation of the terminaltype arterial modifications were successfully embolized. The craniocervical flow modification was feasible. Control angiograms, left CCA injection, showed persistent occlusion of the arterial branches supplying the RM, and control angiograms, right CCA injection, revealed patency of the right $\mathrm{APhAs}$ and improved contrast enhancement of the RM, because of the hemodynamic modifications (Fig. 2).

The feasibility of the endovascular model creation of terminal-type circulation in the right APhAs was evaluated qualitatively by using selective DSA runs postprocedure and quantitatively by comparing the ultrasonography
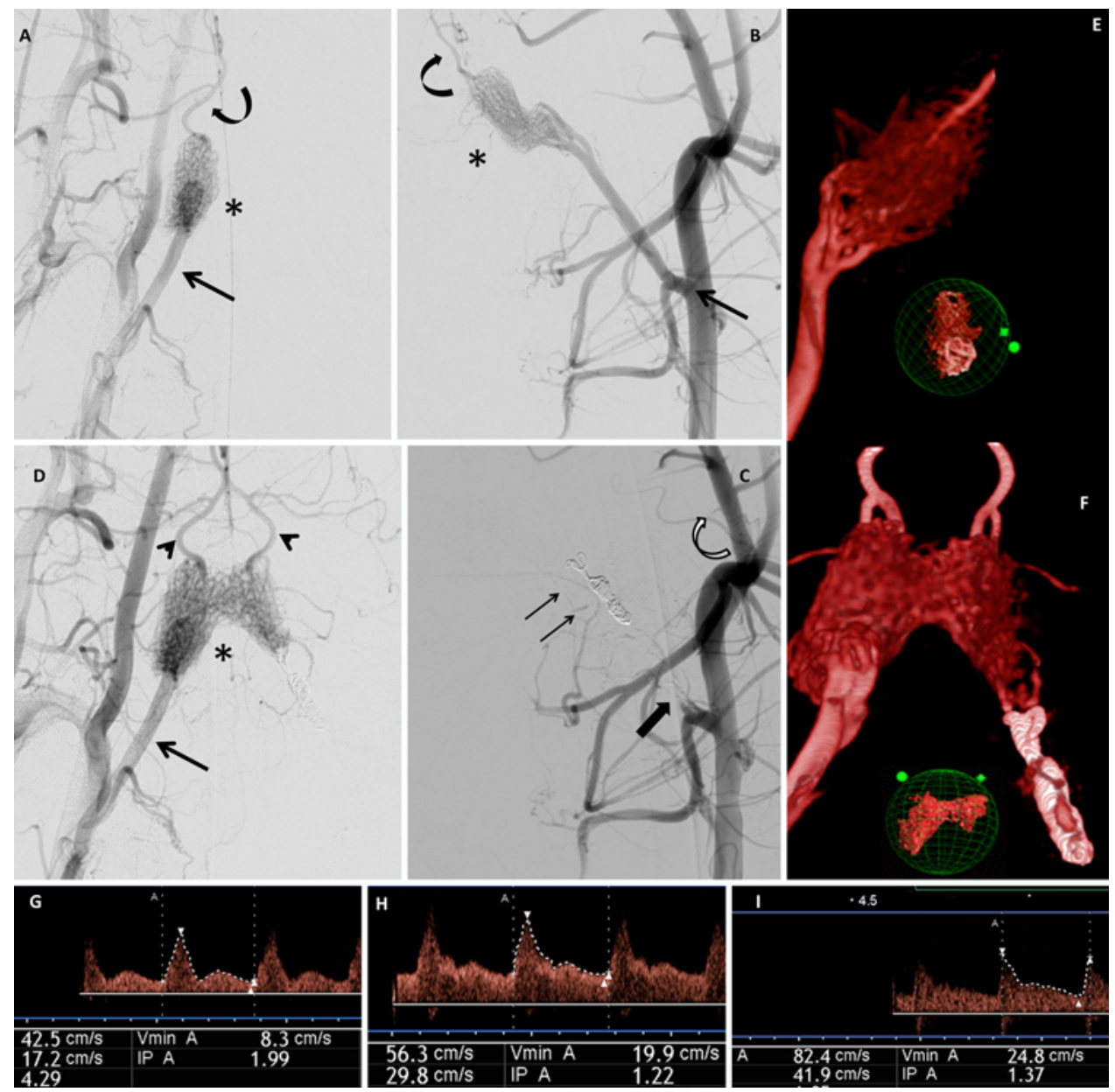

FIG. 2. Endovascular flow modification procedure in Large White swine, aiming to achieve terminal circulation in the right APhA. A: Selective DSA image (working projection), right CCA injection, anteroposterior view, showing the right APhA (straight arrow) and the right lobe of the RM (asterisk). Curved arrow indicates the right brain carotid artery arising from the right lobe of the RM. B: Selective DSA image, left CCA injection, showing the CCA-APhA bifurcation (straight arrow), the left lobe of the RM (asterisk), and the left brain carotid artery (curved arrow). C: Control DSA image, left CCA injection, obtained after selective embolization of the left APhA with coils (thin straight arrows), which resulted in its occlusion (thick arrow). Hyperselective microcatheterization of the arteria anastomotica with a flow-directed microcatheter, followed by biological glue injection at $50 \%$ dilution and taking care to avoid glue diffusion into the RM , resulted in occlusion of the anastomotic ECA network to the RM (curved white arrow). The left lobe of the RM is not visualized by the left CCA injection. D: Control DSA image, right CCA injection, obtained after left APhA occlusion, showing both lobes of the RM (asterisk), both brain carotid arteries (arrowheads), as well as more intense ICA visualization (arrow) than in panel A (before the flow modification procedure). E: Three-dimensional rotational angiography reconstruction, right CCA injection, showing the enhancing part of the RM before the terminal-type endovascular modification. F: Three-dimensional rotational angiography reconstruction, right CCA injection, showing the enhancing part of the RM after the terminal-type endovascular modification; almost double the initial volume is now visible. G-I: Ultrasonographic waveforms obtained in the middle part of the right APhA, from left to right: before the terminal-type endovascular modification, immediately after modification, and at 3 weeks post-modification. Immediately after modification, the blood velocities, which are proportional to the flow rate according to Poiseuille's law, increase significantly since the whole brain supply previously ensured by both APhAs is now coming only from the right side. An additional increase after 3 months shows stability of the terminal type of modification and further reflects the compensatory vasodilation mechanisms and natural animal growth. Figure is available in color online only. 
TABLE 1. Flow model validation*

\begin{tabular}{|c|c|c|c|c|c|}
\hline \multirow[b]{2}{*}{ Parameter } & \multicolumn{3}{|c|}{ APhA Mean Velocity (cm/sec) } & \multirow[b]{2}{*}{$\%$ Change (increase)† } & \multirow[b]{2}{*}{$\%$ Difference } \\
\hline & Before Occlusion & After Occlusion & After 3 Wks of FU & & \\
\hline Subject 1 & 17.2 & 29.8 & 41.9 & 73.25 & 53.62 \\
\hline Subject 2 & 11.8 & 21.3 & 36.4 & 80.50 & 57.40 \\
\hline Arithmetic mean \pm SD & $14.5 \pm 3.8$ & $25.5 \pm 6.0$ & $39.2 \pm 3.9$ & 75.8 & 55 \\
\hline \multicolumn{6}{|c|}{$\begin{array}{l}\text { FU = follow-up; } \mathrm{SD}=\text { standard deviation. } \\
\text { * Mean blood flow velocities were measured using color Doppler ultrasonography before and after endovascular cerebrofacial flow modifica- } \\
\text { tions to produce the terminal type of circulation in the right APhA. } \\
\dagger \text { Calculation of percentage change from before to after occlusion: }[(\mathrm{V} 2-\mathrm{V} 1) /|\mathrm{V} 1|] \times 100 \text {. } \\
\ddagger \text { Calculation of percentage difference from before to after occlusion: }\left[|\Delta \mathrm{V}| /\left(\sum \mathrm{V} / 2\right)\right] \times 100=[|(\mathrm{V} 1-\mathrm{V} 2)| /(\{\mathrm{V} 1+\mathrm{V} 2\} / 2)] \times 100\end{array}$} \\
\hline
\end{tabular}

velocity waveform values before and after embolization. Outcomes are summarized in Table 1. Following the terminal-type flow modification, an increase of $75.8 \%$ in the mean velocity values was observed.

A further increase in velocity values was observed 3 weeks poststenting, which was attributed to the continuous aspiration effect related to the terminal type of circulation. Primary and secondary end points were achieved. The mean RM volume (3D rotational angiography reconstructions, right CCA injection) before vascular modification was $566 \pm 59 \mathrm{ml}(95 \%$ CI 33-1099 ml) and after modification was $1054 \pm 92 \mathrm{ml}(95 \%$ CI 222-1886 ml).

\section{Preliminary Results}

The absence of lesions on diffusion-weighted imaging was confirmed poststenting and on follow-up for all animals. Baseline characteristics of the animals are summarized in Table 2 . The mean case-averaged velocity values before stenting were $12.5 \pm 6.2 \mathrm{~cm} / \mathrm{sec}(95 \% \mathrm{CI}-43.3$ to 68.4) for Group A and $17.8 \pm 5.5 \mathrm{~cm} / \mathrm{sec}(95 \% \mathrm{CI}-32.1$ to 67.7) for Group B. Respective values after stenting were $10.1 \pm 3.1 \mathrm{~cm} / \mathrm{sec}(95 \% \mathrm{CI}-17.1$ to 37.5$)$ and $19.9 \pm 2.9 \mathrm{~cm} /$ sec $(95 \% \mathrm{CI}-6.3$ to 46.1$)$. Mean velocity values immediately poststenting for Group A were reduced (Fig. 3). On the contrary, mean velocity values after stenting did not decrease for Group B (Fig. 4).

Maximal peak systolic velocities for Group A were 18.4 $\pm 10.2 \mathrm{~cm} / \mathrm{sec}(95 \% \mathrm{CI}-72.7$ to 109.5$)$ before stenting and $14.2 \pm 4.5 \mathrm{~cm} / \mathrm{sec}(95 \% \mathrm{CI}-25.7$ to 54.3$)$ after stenting. The respective values for Group B were $23.7 \pm 2.4 \mathrm{~cm} /$ sec (95\% CI 2.1-45.3) and $26.3 \pm 1.9 \mathrm{~cm} / \mathrm{sec}(95 \%$ CI 9.4-
43.2). Maximal velocities increased poststenting in Group B, while they decreased in Group A.

The mean flow rate for Group A was $0.31 \pm 0.19 \mathrm{ml} /$ sec $(95 \% \mathrm{CI}-1.39$ to 2.01$)$ before stenting and $0.21 \pm 0.07$ $\mathrm{ml} / \mathrm{sec}(95 \% \mathrm{CI}-0.45$ to 0.87$)$ after stenting. The mean flow rate for Group B before stenting was $0.87 \pm 0.32 \mathrm{ml} /$ sec $(95 \% \mathrm{CI}-1.98$ to 3.73$)$ and after stenting was $0.76 \pm$ $0.13 \mathrm{ml} / \mathrm{sec}(95 \% \mathrm{CI}-0.41$ to 1.93$)$. A $24.4 \%$ decrease in the mean flow rate was observed after stenting in the anastomotic-type model and a 9.6\% decrease in the terminal-type model. Even though our sample is very small, the statistical comparison of mean flow rates after stenting between the 2 groups revealed a statistically significant difference $(p=0.035$, Welch test).

The mean ostium surface for Group A was 32,776 \pm $15,937 \mu^{2}$ (95\% CI $-110,410$ to 175,962$)$ and for Group B was 422,334 $\pm 12,602 \mu \mathrm{m}^{2}(95 \%$ CI 309,109-535,558). Ostia in Group A were preocclusive, whereas in Group B they were sufficiently patent (Fig. 5). The percentage difference between the mean ostia surfaces of the 2 groups was $171.2 \%$. The power of this preliminary study, calculated using the means and standard deviation of the means of the patent ostia at 3 months, was significant (standardized effect [ES]: -24.4436, Type I error rate [alpha]: 0.0500, critical value of $\mathrm{t}: 4.3027$, power: 1.0000$)$. On the contrary, a larger sample is needed to evaluate velocity changes with more statistical power.

\section{Validity of the Flow Model Regarding Measurable Outcomes}

The MRI protocol was able to detect arithmetic differ-

TABLE 2. Preliminary results

\begin{tabular}{|c|c|c|c|c|c|c|c|c|c|c|}
\hline \multirow[b]{2}{*}{$\begin{array}{l}\text { Case } \\
\text { No. }\end{array}$} & \multirow[b]{2}{*}{ Group } & \multicolumn{2}{|c|}{ Diameter (mm) } & \multirow[b]{2}{*}{$\begin{array}{c}\text { Stent } \\
\text { Size }(\mathrm{mm})\end{array}$} & \multicolumn{5}{|c|}{ APhA Mean Flow Rate } & \multirow{2}{*}{$\begin{array}{c}\text { Ostium } \\
\text { Surface } \\
\text { at } 3 \text { Mos } \\
\left(\mu \mathrm{m}^{2}\right)\end{array}$} \\
\hline & & CCA & ECA & & $\begin{array}{l}\text { Before } \\
\text { Stenting } \\
\text { (cm/sec) }\end{array}$ & $\begin{array}{l}\text { Immediately After } \\
\text { Stenting (cm/sec) }\end{array}$ & $\begin{array}{l}\text { Arithmetic } \\
\text { Difference }\end{array}$ & $\begin{array}{l}\text { \% Change } \\
\text { (decrease) }^{*}\end{array}$ & $\begin{array}{c}\% \\
\text { Difference } \dagger\end{array}$ & \\
\hline 1 & A & 5.01 & 4.9 & $5.00 \times 20$ & 0.445 & 0.266 & 0.179 & 40.22 & 50.35 & 44,045 \\
\hline 2 & A & 4.51 & 4.23 & $4.50 \times 20$ & 0.177 & 0.162 & 0.015 & 8.47 & 8.85 & 21,507 \\
\hline 3 & B & 4.52 & 4.3 & $4.50 \times 20$ & 0.649 & 0.669 & -0.02 & -3.08 & 3.05 & 413,423 \\
\hline 4 & B & 5.04 & 4.65 & $5.00 \times 20$ & 1.098 & 0.853 & 0.245 & 22.31 & 25.11 & 431,245 \\
\hline
\end{tabular}

* Calculation of percentage change in APhA mean flow rate from before stenting to immediately after stenting: [(V2 - V1)/|V1|] × 100. "Decrease" is applicable for all cases except Case 3.

† Calculation of percentage difference in APhA mean flow rate from before stenting to immediately after stenting: $\left[|\Delta \mathrm{V}| /\left(\sum \mathrm{V} / 2\right)\right] \times 100=[\mid(\mathrm{V} 1-$ $\mathrm{V} 2) / /(\{\mathrm{V} 1+\mathrm{V} 2\} / 2)] \times 100$. 

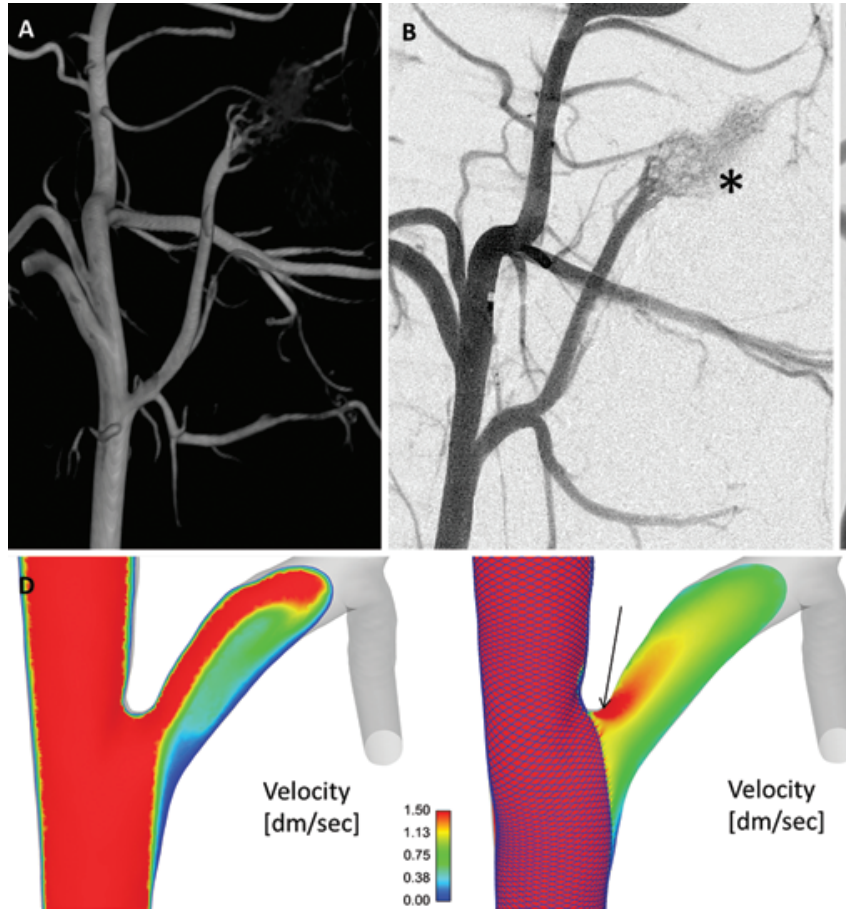
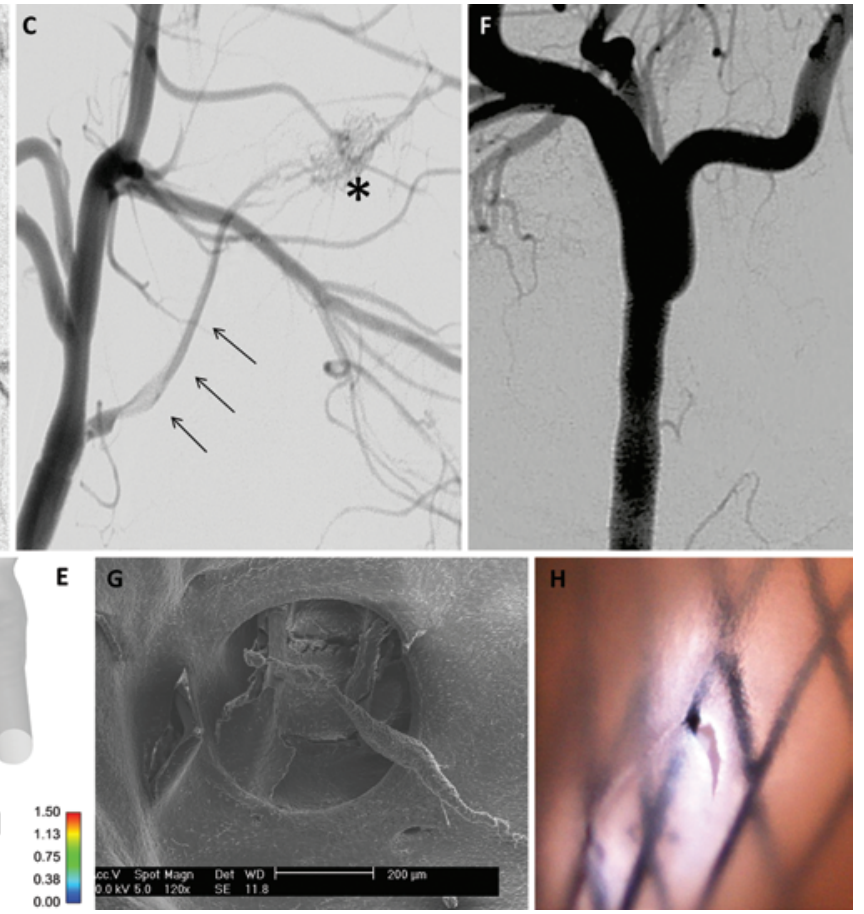

FIG. 3. Representative Group A case, anastomotic type of circulation, DSA studies before and after stenting, as well as at 3 months, with quantitative and qualitative flow analysis. A: Three-dimensional rotational angiography reconstruction of the right CCA, working projection. B: Selective DSA image, right CCA injection, working projection, obtained before stenting, showing the microcatheter placed in the ECA at the level of the deployment starting point. Asterisk indicates the right lobe of the RM. C: Selective DSA run, working projection, obtained right after stenting, showing moderate visualization of the right APhA (arrows). This fact is explained by the presence of collateral supply by the left APhA; thus, flow rate in the right APhA decreases. Poorer visualization of the RM (asterisk) is evident, as compared with its appearance on the prestenting run. D and E: Computational fluid dynamic reconstructions of maximal velocities at the CCA-APhA bifurcation level before and after stenting, respectively. Notice the significant decrease in velocities poststenting, with a minimal region of the stent maintaining high velocity values (arrow, E). F: Three-month follow-up selective DSA image, working projection, showing no visualization of the right APhA. The RM and the brain continued to be supplied by the left side. G: Scanning electron microscopy image of the ostium at 3 month showing a minimal region of ostium persistence; this is a preocclusive state. $\mathrm{H}$ : Optical microscopic image of the same ostium featured in panel $\mathrm{G}$, obtained after chemical fixation and before critical point drying, showing an endothelial lining that is about to occlude the last patent pore of the stent. Figure is available in color online only.

ences in mean flow rates before and after stenting with precision, with values starting at $-0.02 \mathrm{ml} / \mathrm{sec}$ (ranging up to $0.245 \mathrm{ml} / \mathrm{sec}$ ) and percentage differences ranging from $3 \%$ to $50 \%$ in the preliminary study. The mean flow rate decrease between the anastomotic and terminal circulation groups (A and B) was easily detectable and significant (24.4\% vs $9.6 \%$, respectively), which further confirms the validity of the flow model. Overall flow modifications were detectable in all cases and were expressed differently between the 2 groups, with a significant reduction after stenting in the anastomotic type of circulation (Group A) and mainly preservation of values in the terminal type of circulation (Group B).

\section{A Posteriori Evaluation of CFD Simulations}

Computational fluid dynamic simulations demonstrated sufficiently the velocity and flow rate modifications measured using time-resolved 3D pcMRA, as discussed above. Qualitative assessment showed good correlation of the CFD simulations with the 30 images/sec selective DSA findings. The measurements of stent strut angles cor- related well with the virtual deployment simulations in all cases (Table 3 and Fig. 6).

\section{Computational Fluid Dynamics Results}

For Group B cases, flow rates and mean velocity values poststenting were maintained, with a large area of the stented ostia maintaining maximal peak systolic velocities, as compared with prestenting values. Pressure gradients through the stent were also maintained.

The pressure gradient after stenting decreased for Group A cases. Velocity and mean flow rate values also showed a significant decrease; the areas of the stented ostia maintaining relatively high maximal velocities and adequate mean flow rates were especially limited. This result correlated with the small areas of patent ostia on the 3-month angiographic and SEM controls. These areas seemed to correspond to the distal part of the ostia (Figs. 3 and 4).

\section{Discussion}

The increasing tendency to use flow diverters in off- 

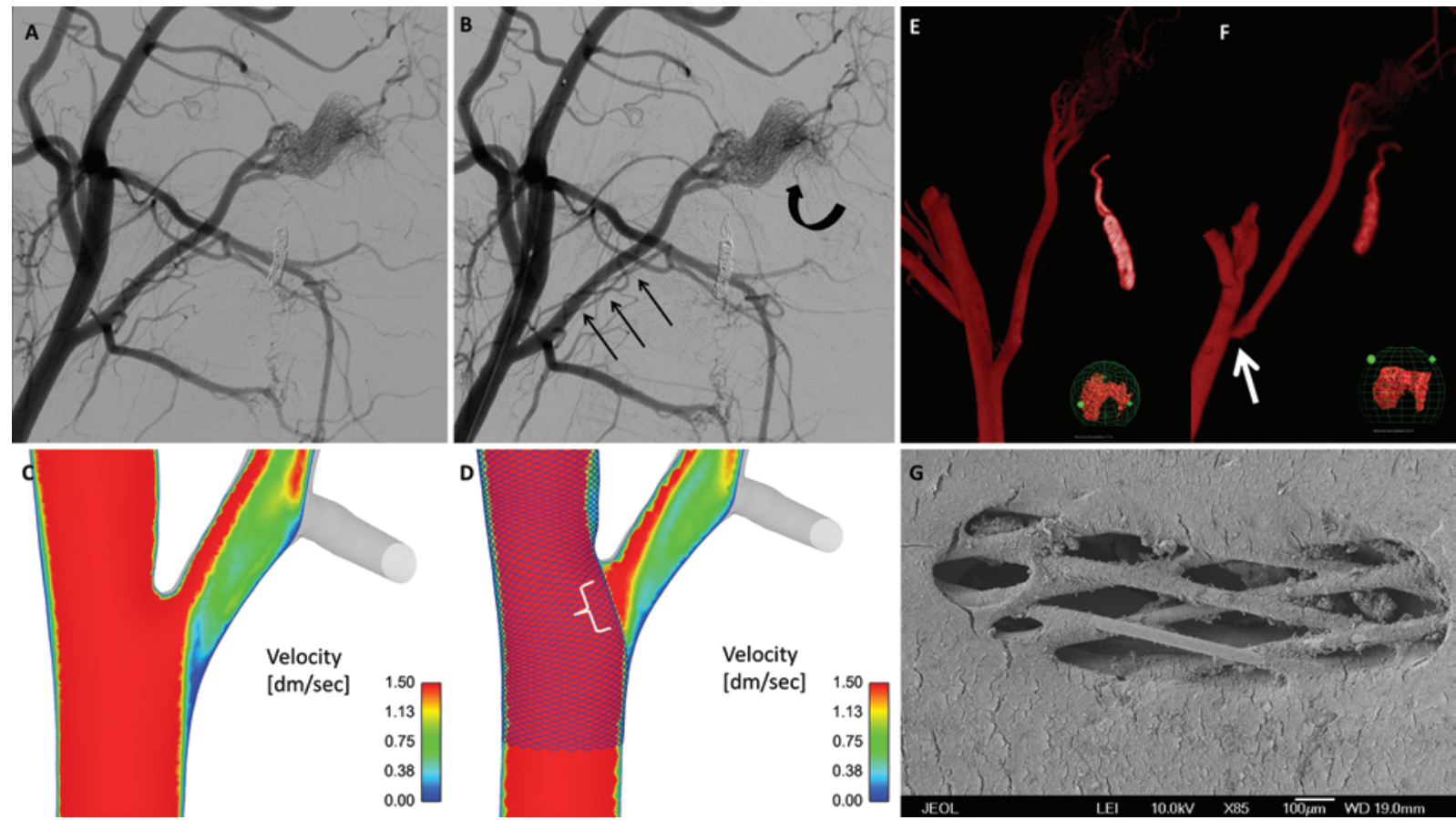

FIG. 4. Representative Group B case, terminal type of circulation, DSA studies before and after stenting with quantitative and qualitative flow analysis. A: Selective DSA run, right CCA injection, working projection, obtained before stenting. B: Selective DSA run, right CCA injection, obtained directly after stenting, showing good visualization of the right APhA (straight arrows) and increased visualization of the RM (curved arrow). This fact is explained by the absence of collateral supply by the left APhA; thus, the flow rate in the right APhA persists, continuing to supply the RM and through it, the brain. $\mathrm{C}$ and $\mathrm{D}$ : Computational fluid dynamic reconstructions of maximal velocities at the CCA-APhA bifurcation level before and after stenting, respectively. Velocity values after stenting persist, and there is an even larger area of high velocity passage (white bracket) through the stent in the poststenting reconstruction. E and F: Three-dimensional rotational angiography reconstructions, right CCA injection, obtained before stenting and at 3 months' angiographic control, respectively, showing only minimal vascular remodeling of the right APhA ostium. G: Scanning electron microscopy image of the stented ostium at 3 months showing a sufficiently patent ostium. Notice the endothelial cells wrapped around the stent struts. Original magnification $\times 85$. Figure is available in color online only.

label arterial sites, ${ }^{7}$ mainly in distal intracranial locations, often involves jailing of intracranial arterial branches with uncertain results in terms of patency. In a recent retrospective case series, during the treatment of 66 aneurysms, 68 uninvolved side branches were covered..$^{11}$ While retrospective clinical studies on jailed arteries are ethically acceptable, a prospective, comparative clinical study could be

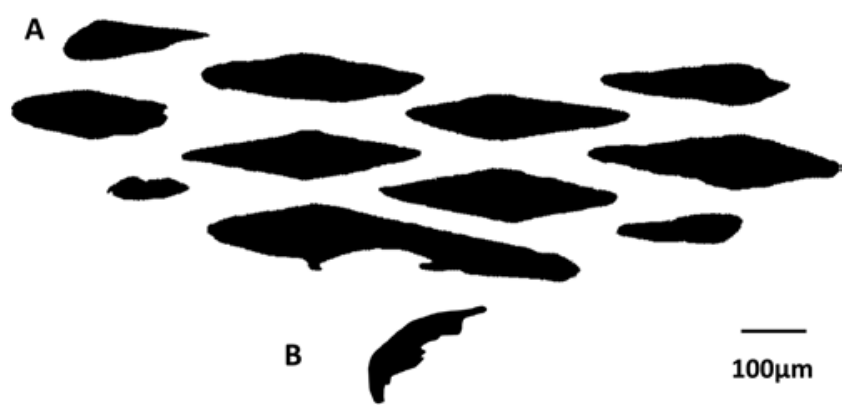

FIG. 5. Graphic representation of the terminal circulation ostium (A), as in the case represented in Fig. 4, compared to the anastomotic circulation ostium featured in Fig. 3 (B). Black represents the regions of patency of the ostia. Notice the important difference between the 2 areas. In the flow competition case, the ostium is around 1/19th the surface of the terminal circulation ostium. Absolute values for these cases were 21,507 $\mu \mathrm{m}^{2}$ and $413,423 \mu \mathrm{m}^{2}$, respectively. problematic since the danger of inadvertent branch occlusion exists, even in adequately premedicated patients. ${ }^{20}$

Apart from a few animal studies, ${ }^{17,18}$ which have reported the patency of jailed arteries after stenting, there are no exhaustive animal data dedicated to jailed branch fate after flow-diverting stenting. The present study is the first to propose an animal model capable of simulating terminal and anastomotic arterial circulation and to provide quantifiable data for FDS research. Distinguishing arterial

TABLE 3. Angle measurements obtained from optical microscopy compared with those obtained with CFD*

\begin{tabular}{ccc}
\hline Measurement No. & OM Angles & CFD Angles \\
\hline 1 & $52.4^{\circ}$ & $44.1^{\circ}$ \\
\hline 2 & $53.7^{\circ}$ & $48.8^{\circ}$ \\
\hline 3 & $58.8^{\circ}$ & $53.2^{\circ}$ \\
\hline 4 & $116.6^{\circ}$ & $124.4^{\circ}$ \\
\hline 5 & $125.9^{\circ}$ & $131.7^{\circ}$ \\
\hline 6 & $133.2^{\circ}$ & $134.7^{\circ}$ \\
\hline
\end{tabular}

$\mathrm{OM}=$ optical microscopy.

* Example from the case shown in Fig. 4, showing acceptable stent representation. None of the measurements differed more than $10^{\circ}$ higher. The comparisons referred to either blunt or acute angles among them. 

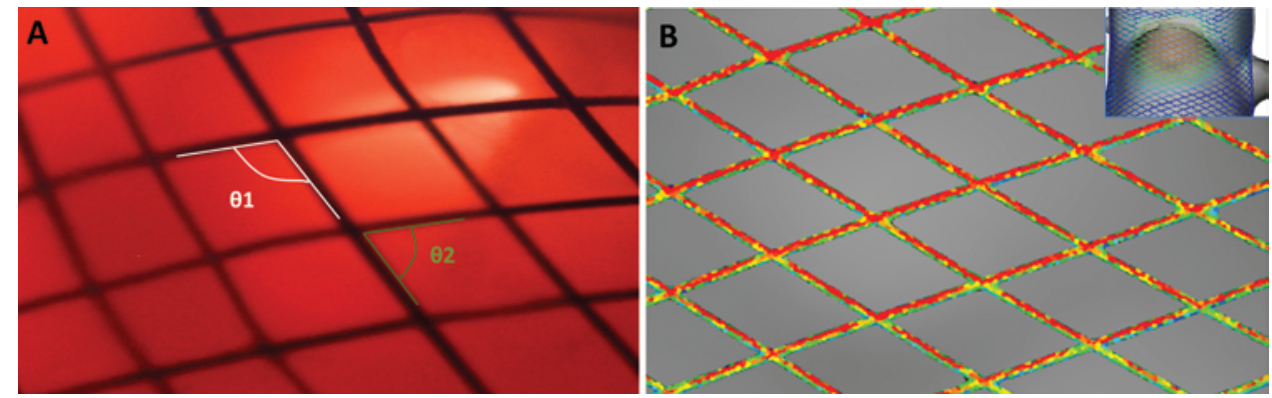

FIG. 6. A: Optical microscopy image of a harvested and chemically fixed (5\% glutaraldehyde in Sorensen's solution) carotid artery. The artery has been longitudinally cut, and the inner surface is exposed. For this image, lighting below the specimen was preferred to make the stent struts appear more clearly and thus allow us to calculate the stent strut angles. The stent is braided, which means that the struts can move freely during positioning; this, in turn, can result in variations in the blunt ( $(\theta 1)$ and acute $(\theta 2)$ angles of the cells. Original magnification $\times 40$. B: Virtual stent deployment in volume meshes reconstructed from the 3D rotational angiography images during the stenting procedure in the same animal. To generate the virtual deployment, the volume element numbers ranged from 10 to 15 million. Three measurements for each type of angle were performed both in the optical and in the virtual images of the stent; the comparison revealed good correlation between the optical images and the virtual stenting (Table 3). Figure is available in color online only.

branches according to the presence or absence of direct collateral flow, with a protocol that provides the possibility of quantitatively measuring the hemodynamic changes, has never been proposed to date.

In the current study we proposed a valid animal flow model for the evaluation of side arterial branches jailed by FDSs, simulating terminal and anastomotic circulation. In clinical practice, the presence or absence of flow competition regarding jailed intracranial arteries seems to play a role in the remodeling process as well as the subsequent patency or occlusion of these branches in sufficiently antiaggregated patients. ${ }^{256}$

This study provides preliminary evidence that, in the presence of flow competition, the decrease in flow rates and velocities after stenting favors significant endothelialization of the covered ostia, potentially leading to branch occlusion. In contrast, the absence of flow competition seems to promote patency of the branch by restricting endothelial coverage through an increase in peak systolic velocities and the persistence of significant flow rates after stenting.

The anastomotic type of circulation (Group A) in this study approximates clinical cases involving jailing of the anterior communicating, posterior communicating, anterior choroidal, and ophthalmic arteries. We demonstrated that, when direct anastomotic circulation is present, jailed branches undergo significant flow rate and velocity reductions, factors that seem to be correlated to significant ostia endothelialization. These conclusions may explain why in direct anastomotic configurations at the level of the circle of Willis in humans, jailed branches occlude without clinical consequence, as confirmed by recent clinical data. ${ }^{4,13}$

The terminal type of circulation (Group B) in this study simulates clinical situations in which direct collateral supply is absent. Such cases are usually encountered in middle cerebral artery (MCA) branches, $\mathrm{A}_{1}-\mathrm{A}_{2}$ aneurysms with a hypoplastic contralateral $\mathrm{A}_{1}$ segment, or the fetal configuration of the posterior cerebral artery. In the absence of flow competition, the jailed branch is expected to more or less maintain its prestenting flow rates and velocities, which in turn results in patency. In clinical studies, late transient symptoms in jailed MCA branches and late-oc- curring, silent diffusion-weighted imaging findings have been described. ${ }^{12,25}$ These events were probably related to pial anastomoses development during the postprocedural period, ${ }^{25}$ resulting in a limited degree of flow competition.

Regarding technical matters in the presented model, the swine RM, which has been successfully used in arteriovenous malformation animal models, ${ }^{8,21}$ was exploited here to obtain direct anastomotic circulation and to accentuate the differences between terminal and anastomotic circulation. The goal was to produce quantifiable, measurable flow rate differences between terminal and anastomotic configurations when an FDS was superimposed at APhA ostia.

Even though swine APhAs are extracranial and thus not directly comparable to human intracranial arteries, they exhibit low-resistance color Doppler arterial flow waveforms similar to those in human intracranial arteries, ${ }^{6}$ providing a more reliable approximation to reality in terms of the blood flow behavior as compared with rabbit abdominal aortas, the most commonly used model to date.

Even though mean velocity increases around $20 \%$ after occlusion of contralateral carotid arteries in animals have been reported in the literature, ${ }^{10}$ a higher end point of at least $40 \%$ was chosen to validate our flow model. This choice was mainly based on the working hypothesis that blood flow rates and volumes should remain adequate for normal brain function after contralateral side arterial occlusions.

Most of the reluctance regarding the clinical use of FDSs in distal intracranial locations comes from the fact that the jailed branch fate remains unclear. The flow competition concept could facilitate therapeutic decision making in challenging intracranial aneurysm cases. The preliminary results presented here can be used as input for a priori power analysis in additional animal studies since statistical power is important to ensure the validity of these observations. The results will be helpful to the neurointerventionist in defining clearer indications for when a jailed branch should or should not be covered in clinical practice, through an a priori qualitative and quantitative analysis of DSA and MRI data before treatment. 


\section{Study Limitations}

This is a feasibility study aimed at providing preliminary data for further research. As such, it lacks a significant number of subjects and thus statistical power. In compliance with ethical standards, an a priori power analysis is needed to avoid unnecessary use of animals in research. The present study allows for the calculation of the necessary number of subjects to further confirm the findings of this preliminary study. Nevertheless, it provides useful data for further research planning and highlights several interesting aspects of the flow changes induced in terminal and anastomotic types of circulation. The results apply to arteries around $1-2 \mathrm{~mm}$ in diameter and thus exclude from generalizations those arteries with diameters in the range of human intracranial perforators. Even though APhAs are not intracranial arteries and thus are not directly comparable to intracranial arteries, especially with respect to smooth muscle adrenergic receptors and endothelial tight junctions, they do provide a reliable approximation of reality in terms of hemodynamics.

\section{Conclusions}

The terminal-type of arterial modification in swine $\mathrm{APhAs}$ is feasible. The flow model is effective for simulating and quantifying terminal and anastomotic arterial circulation. After jailing branches with flow diverters, sufficient patency of the branches was observed in the absence of flow competition. In contrast, preocclusive stenoses were observed with the anastomotic type of circulation. Smaller differences in flow rates before and after stenting also seemed to be correlated with the terminal type of circulation. Further research exploiting this model is needed to confirm these preliminary results.

\section{Acknowledgments}

The study was partially financed by the University of Limoges, France (www.unilim.fr), which provided the animal acquisition and care; and the Federal Ministry of Education and Research in Germany within the Research Campus STIMULATE under Grant No. 03FO16102A.

\section{References}

1. Becske T, Kallmes DF, Saatci I, McDougall CG, Szikora I, Lanzino G, et al: Pipeline for uncoilable or failed aneurysms: results from a multicenter clinical trial. Radiology 267:858868,2013

2. Berg P, Janiga G, Beuing O, Rössl C, Thévenin D: Virtual intracranial stenting: how does stent porosity influence hemodynamics? Interventional Neuroradiol 19:191, 2013 (Abstract)

3. Berg P, Stucht D, Janiga G, Beuing O, Speck O, Thévenin D: Cerebral blood flow in a healthy Circle of Willis and two intracranial aneurysms: computational fluid dynamics versus four-dimensional phase-contrast magnetic resonance imaging. J Biomech Eng 136:136, 2014

4. Brinjikji W, Lanzino G, Cloft HJ, Kallmes DF: Patency of the posterior communicating artery after flow diversion treatment of internal carotid artery aneurysms. Clin Neurol Neurosurg 120:84-88, 2014

5. Brinjikji W, Murad MH, Lanzino G, Cloft HJ, Kallmes DF: Endovascular treatment of intracranial aneurysms with flow diverters: a meta-analysis. Stroke 44:442-447, 2013
6. Cebral JR, Raschi M, Mut F, Ding YH, Dai D, Kadirvel R, et al: Analysis of flow changes in side branches jailed by flow diverters in rabbit models. Int J Numer Methods Biomed Eng 30:988-999, 2014

7. Chalouhi N, Starke RM, Yang S, Bovenzi CD, Tjoumakaris $\mathrm{S}$, Hasan D, et al: Extending the indications of flow diversion to small, unruptured, saccular aneurysms of the anterior circulation. Stroke 45:54-58, 2014

8. Chaloupka JC, Viñuela F, Robert J, Duckwiler GR: An in vivo arteriovenous malformation model in swine: preliminary feasibility and natural history study. AJNR Am J Neuroradiol 15:945-950, 1994

9. Chang C, Uchiyama A, Ma L, Mashimo T, Fujino Y: A comparison of the effects on respiratory carbon dioxide response, arterial blood pressure, and heart rate of dexmedetomidine, propofol, and midazolam in sevofluraneanesthetized rabbits. Anesth Analg 109:84-89, 2009

10. García-Villalón AL, Roda JM, Alvarez F, Gómez B, Diéguez G: Carotid blood flow in anesthetized rats: effects of carotid ligation and anastomosis. Microsurgery 13:258-261, 1992

11. Gascou G, Lobotesis K, Brunel H, Machi P, Riquelme C, Eker O, et al: Extra-aneurysmal flow modification following pipeline embolization device implantation: focus on regional branches, perforators, and the parent vessel. AJNR Am J Neuroradiol 36:725-731, 2015

12. Iosif C, Camilleri Y, Saleme S, Caire F, Yardin C, Ponomarjova S, et al: Diffusion-weighted imaging-detected ischemic lesions associated with flow-diverting stents in intracranial aneurysms: safety, potential mechanisms, clinical outcome, and concerns. J Neurosurg 122:627-636, 2015

13. Iosif C, Carles P, Trolliard G, Yardin C, Mounayer C: Scanning electron microscopy for flow-diverting stent research: technical tips and tricks. Microscopy (Oxf) 64:219-223, 2015

14. Janiga G, Berg P, Beuing O, Neugebauer M, Gasteiger R, Preim B, et al: Recommendations for accurate numerical blood flow simulations of stented intracranial aneurysms. Biomed Tech (Berl) 58:303-314, 2013

15. Janiga G, Rössl C, Skalej M, Thévenin D: Realistic virtual intracranial stenting and computational fluid dynamics for treatment analysis. J Biomech 46:7-12, 2013

16. Jiang J, Strother C, Johnson K, Baker S, Consigny D, Wieben $\mathrm{O}$, et al: Comparison of blood velocity measurements between ultrasound Doppler and accelerated phase-contrast MR angiography in small arteries with disturbed flow. Phys Med Biol 56:1755-1773, 2011

17. Kallmes DF, Ding YH, Dai D, Kadirvel R, Lewis DA, Cloft HJ: A new endoluminal, flow-disrupting device for treatment of saccular aneurysms. Stroke 38:2346-2352, 2007

18. Kallmes DF, Ding YH, Dai D, Kadirvel R, Lewis DA, Cloft HJ: A second-generation, endoluminal, flow-disrupting device for treatment of saccular aneurysms. AJNR Am J Neuroradiol 30:1153-1158, 2009

19. Kilkenny C, Browne W, Cuthill IC, Emerson M, Altman DG: Animal research: reporting in vivo experiments-the ARRIVE guidelines. J Cereb Blood Flow Metab 31:991993, 2011

20. Lall RR, Crobeddu E, Lanzino G, Cloft HJ, Kallmes DF: Acute branch occlusion after Pipeline embolization of intracranial aneurysms. J Clin Neurosci 21:668-672, 2014

21. Massoud TF, Ji C, Viñuela F, Guglielmi G, Robert J, Duckwiler GR, et al: An experimental arteriovenous malformation model in swine: anatomic basis and construction technique. AJNR Am J Neuroradiol 15:15371545,1994

22. Newcomer CE: The evolution and adoption of standards used by AAALAC. J Am Assoc Lab Anim Sci 51:293-297, 2012

23. Ohlsson A, Fong K, Ryan ML, Yap L, Smith JD, Shennan AT, et al: Cerebral-blood-flow-velocity measurements in 
neonates: technique and interobserver reliability. Pediatr Radiol 21:395-397, 1991

24. Oliveira JC, Campos R: A systematic study of brain base arteries in the wild boar (Sus scrofa scrofa). Anat Histol Embryol 34:232-239, 2005

25. Saleme S, Iosif C, Ponomarjova S, Mendes G, Camilleri Y, Caire F, et al: Flow-diverting stents for intracranial bifurcation aneurysm treatment. Neurosurgery 75:623-631, 2014

\section{Disclosures}

Dr. Mounayer is a scientific consultant for Covidien-Medtronic. The study was partially financed by Covidien-Medtronic (www. covidien.com), which provided the neurovascular material used.

\section{Author Contributions}

Conception and design: Iosif, Mounayer. Acquisition of data: Iosif, Berg, Carles, Pedrolo-Silveira, Mendes, Waihrich, Couquet, Yardin. Analysis and interpretation of data: Iosif, Berg. Drafting the article: Iosif. Critically revising the article: Ponsonnard, Saleme, Trolliard, Yardin, Mounayer. Reviewed submitted version of manuscript: Iosif, Yardin. Approved the final version of the manuscript on behalf of all authors: Iosif. Statistical analysis: Iosif. Administrative/technical/material support: Ponsonnard. Study supervision: Mounayer.

\section{Correspondence}

Christina Iosif, 2 Avenue Martin Luther King, Limoges 87042, France. email: christina.iosif@gmail.com. 